\title{
Incoherent microwave-induced resistive states of small Josephson junctions
}

\author{
Y. Koval \\ Physikalisches Institut III, Universität Erlangen-Nürnberg, Erlangen D-91058, Germany \\ M.V. Fistul \\ Theoretische Physik III, Ruhr-Universität Bochum, Bochum D-44801, Germany \\ A.V. Ustinov \\ Physikalisches Institut, Karlsruhe Institute of Technology, Karlsruhe D-76131, Germany \\ E-mail: alexey.ustinov@pi.uni-karlsruhe.de
}

Received May 7, 2010

\begin{abstract}
We report an experimental and theoretical study of low-voltage resistive states that are observed in small tunnel Josephson junctions under microwave radiation. The studied features emerge from Shapiro steps on the current-voltage characteristics and appear when both thermal fluctuations and high frequency dissipation are strong. In the absence of microwave radiation Josephson junctions display under these conditions a phase diffusion supercurrent branch characterized by a finite small resistance and hysteretic switching to high voltage range. As the microwave radiation is applied, we experimentally observe three different types of resistive states in the currentvoltage characteristics. First, the phase diffusion branch steadily evolves and its maximum reached voltage $V_{m}$ increases with the microwave power. Another interesting observed feature is a zero-crossing resistive state characterized by a negative resistance. Finally, we find that a low-voltage resistive state can split in numerous hysteretic fine branches resembling incoherent Shapiro-like steps. The appearance of a particular resistive state depends on an interrelation between the Josephson energy $E_{J}$, energy of thermal fluctuations $k_{B} T$, and the frequency of microwave radiation $\omega$. Our theoretical analysis based on an incoherent multi-photon absorption of a junction biased in the Josephson phase diffusion regime, is in a good accord with experimental observations.
\end{abstract}

PACS: 74.50.+r Tunneling phenomena; point contacts, weak links, Josephson effects;

74.40.-n Fluctuation phenomena;

74.78.Na Mesoscopic and nanoscale systems.

Keywords: low-voltage resistive states, Josephson junctions, microwave radiation, thermal fluctuations, high frequency dissipation.

\section{Introduction}

Small Josephson tunnel junctions show numerous versatile and fascinating phenomena ranging from Coulomb blockade of Cooper pair transport to macroscopic quantum tunneling [1,2]. These phenomena can be observed under extremely low temperatures and small dissipation. However, even in the opposite limit of moderate temperatures and a substantial frequency-dependent damping, same small tunnel junctions display an interesting incoherent regime of a Josephson phase diffusion [3-5].

The regime of Josephson phase diffusion can be viewed as a classical phenomenon, and it occurs as a peculiar in-

(c) Y. Koval, M.V. Fistul, and A.V. Ustinov, 2010 terplay between thermal fluctuations, Josephson coupling and dissipation. The Josephson phase diffusion manifests itself by a specific resistive state with (typically extremely small) resistance depending on the bias current. Here, the «true» superconducting state is absent and there is a small voltage drop at non-zero current flowing through the junction. Moreover, in spite of the finite resistance of this supercurrent branch the current-voltage characteristics ( $I-V$ curves) display the hysteretic behavior when the switching to the region of large voltages. This hysteresis originates from the difference between low-frequency and high-frequency damping. This dynamic regime provides an extremely nonlinear $I-V$ curve, and the low-voltage 
phase-diffusion branch of this curve is characterized by maximum reached voltage $V_{m}$ and current $I_{m}$. These both parameters depend on temperature and the junction size. Typically, Josephson junctions that display such behavior have sub-micrometer size.

Due to the fact that a small junction biased in Josephson phase diffusion regime represents itself an extremely nonlinear system, it is quite natural and rather interesting to investigate and understand what happens in this system under an external ac excitation, e.g. when the junction is irradiated by microwaves. In our first paper published few years ago [6] we have studied experimentally and theoretically the enhancement of phase diffusion by microwaves. It has been observed that the application of microwaves leads to a shift of the phase diffusion branch to larger voltages, and the zero-voltage resistance substantially increases. Moreover, we have mentioned that the resistive state with a negative resistance can be also observed, but no detailed experimental or theoretical study was provided at that time.

It is worth mentioning another example from very different field of semiconductor superlattices [7] and two-dimensional electron gas structures. In external magnetic field [8] the application of a microwave radiation can lead to appearance of resistive states that qualitatively differ from the autonomous (i.e., observed in the absence of ac field) ones. In particular, some of these cases have shown a negative resistance. These phenomena are related to the subject of this paper and originate from a process of incoherent multi-photon absorption by a nonlinear system.

In this paper, we present an experimental and theoretical study of microwave induced low-voltage resistive states of small Josephson tunnel junctions. In particular, we focus on the crossover regime between completely incoherent microwave-assisted phase diffusion [6] and the conventional textbook-like Shapiro steps [9]. Our experimental data are compared with the theoretical analysis, which consistently explains an enhanced phase diffusion branch [6], the zero-crossing branch characterized by a negative resistance, and a fine-structure branching of the resistive state. We also show how various parameters such as the temperature, junction size and frequency of microwave radiation influence the $I-V$ curve. All our experimental results are well explained by the model of incoherent multi-photon absorption in small Josephson junction biased in Josephson phase diffusion regime.

In Sec. 2, we discuss in detail our method for the fabrication of sub-micron size Josephson tunnel junctions. The details of the experimental setup are also presented. The first part of Sec. 3 illustrates the observed current-voltage characteristics of various small Josephson junctions subject to the external microwave irradiation. In the second part of Sec. 3 we present our theoretical analysis of diverse microwave induced low-voltage resistive states. The Sec. 4 contains the discussion and conclusions.

\section{Sample preparation and experimental details}

For the reported experiments, we have fabricated several sub-micron size $\mathrm{Nb} / \mathrm{AlO}_{x} / \mathrm{Nb}$ Josephson tunnel junctions. The schematic of the sample preparation is shown in Fig. 1. We used three-layer of $\mathrm{Nb} / \mathrm{AlO}_{x} / \mathrm{Nb}$, which is deposited on a thermally oxidized Si wafer (see Fig. 1,a). The critical current density of the trilayer is about $200 \mathrm{~A} / \mathrm{cm}^{2}$. By making use of electron-beam lithography and reactive ion etching (RIE), junctions of different areas are defined. The RIE process is performed in $\mathrm{CF}_{4} / \mathrm{O}_{2}$ mixture. Immediately after RIE, in the same setup, the $\mathrm{O}_{2}$ plasma is used to anodize the side walls of mesas and to remove the rest of resist. The obtained structure is shown schematically in Fig. $1, b$.

As the next step the pattering of the bottom $\mathrm{Nb}$ layer is carried out by using the lithography and RIE. Niobium is removed from everywhere on the substrate except of the place where the wiring to the bottom part of the Josephson junction locates (see Fig. 1,c). For insulation of the side walls of Josephson junctions and the area around them, we used cross-linked polymethylmethacrylate (PMMA) [10]. This material is a well known for many years as an electron resist, that can behave as a positive one at low-dose irradiation and as a negative one at high-dose irradiation. Such a property of PMMA we use in our fabrication method. PMMA with molecular weight $950 \mathrm{~K}$ was spanned onto the substrate from a liquid solution in chlorbenzene (Fig. 1,d). By making use of the electron-beam lithography, the windows in the top $\mathrm{Nb}$ layer located above patterned Josephson junctions are opened (Fig. 1,e). The following high-dose electron exposure and development leave the cross-linked PMMA only around Josephson junctions (Fig. 1,f).

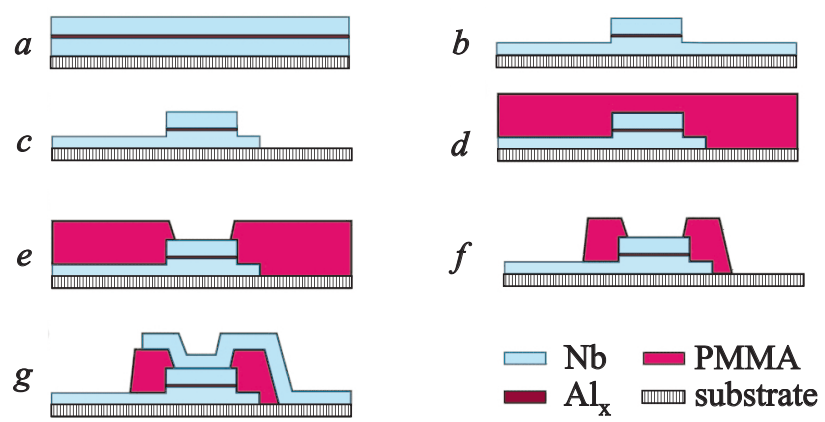

$h$

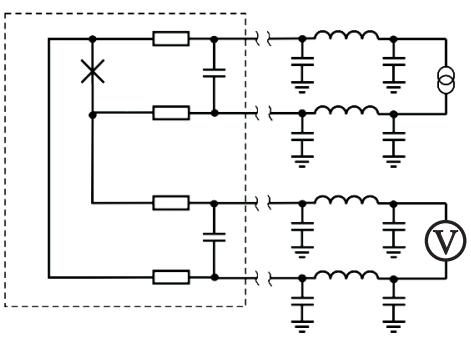

Fig. 1. The steps of samples fabrications $(a-g)$ and the electrical scheme of measurements $(h)$. 
As the last step we formed wiring electrodes to the top layer of Josephson junctions (see Fig. 1,g). Nb layer is deposited by sputtering. Again the electron-beam lithography and RIE are used for patterning of the wiring layer. By the described above method, we prepared junctions of different area from $\simeq 0.23 \times 0.23 \mu \mathrm{m}$ to $\simeq 2 \times 2 \mu \mathrm{m}$.

We measured dc current-voltage characteristics $(I-V$ curves) of Josephson junctions at different temperatures. It is a well known that $I-V$ curves of a small Josephson junction are extremely sensitive to fluctuations, e.g. a current noise. Thus, to diminish the current noise influence on the $I-V$ curves, the wires for electric current biasing and for voltage measurement are filtered according to the scheme shown in Fig. 1, $h$. For this purpose we used Pifilters with cut-off frequency $18 \mathrm{GHz}$. These filters are mounted approximately $1 \mathrm{~m}$ away from the sample and during the measurements are at room temperature. In addition, the cold $R-C$ filters are placed approximately $5 \mathrm{~mm}$ away from the junctions, and stayed at the sample's temperature during the measurements. The cold filters consisted of surface mounted resistors and ceramic capacitors. At room temperature their nominal values are $103 \Omega$ and $0.1 \mu \mathrm{F}$, correspondingly. Microwave radiation is applied by inductively coupled antenna placed at the distance $5 \mathrm{~mm}$ from the sample surface.

The measurements of $I-V$ curves at $4.2 \mathrm{~K}$ are performed in liquid ${ }^{4} \mathrm{He}$. Further measurements at variable temperatures are done in ${ }^{3} \mathrm{He}$ cryostat. The lowest temperature reported in our experiments presented below was $0.3 \mathrm{~K}$.

\section{Microwave induced current-voltage characteristics: experiment}

First, we measured $I-V$ curves of small Josephson junctions of different areas in the absence of microwave radiation. The typical $I-V$ curve is shown in Fig. 2 . The $I-V$ curve displays the low-voltage resistive state corres-

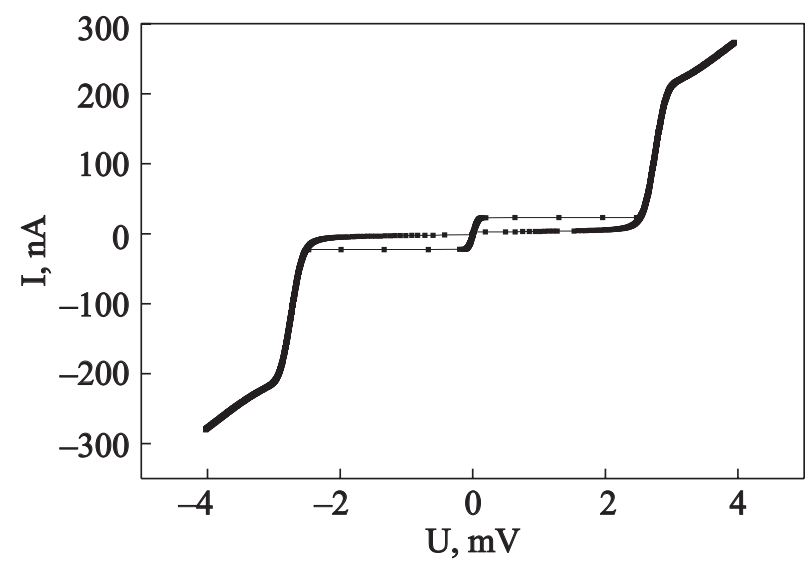

Fig. 2. The typical $I-V$ curve of a Josephson junction biased in the Josephson phase diffusion regime. The area of Josephson junction is $0.28 \times 0.28 \mu \mathrm{m}$, and the temperature is $4.2 \mathrm{~K}$. ponding to the Josephson phase diffusion regime and a hysteretic switching to the high-voltage region. The observed hysteresis is rather large, i.e. the retrapping current $I_{\text {ret }} \ll I_{m}$, and it is a consequence of a large quasiparticle resistance of a Josephson junction.

The experimentally observed parameters of all junctions of different areas are summarized in Table 1. The nominal critical current $I_{c}$ was determined by two methods: taken as a half of the current rise at the gap voltage $V=2 \Delta / e$ or the maximum expected from AmbegaokarBaratoff critical current $I_{\mathrm{A} B}=\pi \Delta /\left(2 e R_{N}\right)$, where $R_{N}$ is the Josephson junction resistance in the normal state. Both methods result to similar values. The nominal critical current and correspondingly the Josephson energy $E_{J}$ varies proportional to the junction area. Notice here, that the maximum reached value of the voltage $V_{m}$ (see Fig. 2) decreases with increasing the junction area, and the two last samples of a large area shown in the Table 1, do not display the phase diffusion branch at all.

Table 1. Parameters of Josephson junctions of different area. All data are obtained at $4.2 \mathrm{~K}$.

\begin{tabular}{c|c|c|c}
\hline \hline Area, $\mu \mathrm{m}^{2}$ & $I_{c}, \mu \mathrm{A}$ & $I_{\text {retrap }}, \mathrm{nA}$ & $R_{N}, \mathrm{k} \Omega$ \\
\hline \hline $0.23 \times 0.23$ & 0.11 & 2.2 & 14.5 \\
\hline $0.28 \times 0.28$ & 0.16 & 6 & 10 \\
\hline $0.47 \times 0.47$ & 0.44 & 15 & 3.5 \\
\hline $1.1 \times 1.1$ & 2.45 & 126 & 0.617 \\
\hline $2 \times 2$ & 8.5 & 410 & 0.176 \\
\hline \hline
\end{tabular}

Next, we applied microwave radiation to the particular Josephson junction of $0.28 \times 0.28 \mu \mathrm{m}$ area and measured the $I-V$ curves on different temperatures. These results, i.e. the dependence of the $I-V$ curves on the power of microwave radiation, are presented in Fig. 3. Notice here that for these particular measurements the large frequency of microwave radiation was used ( $\omega=20 \mathrm{GHz}$ ).

We observed that in the absence of microwave radiation the Josephson phase diffusion is diminished, and therefore, both the characteristic voltage $V_{m}$ and the zero-voltage resistance were strongly suppressed as the temperature is decreased (see Fig. 3). In the presence of microwave radiation we observed a rather unusual low-voltage resistive state [6]. Thus, at large temperature, e.g. at $T=5 \mathrm{~K}$, an increase of a power of microwave radiation leads to a strong increase of zero-voltage resistance, and shifts the voltage $V_{m}$ to higher values (see Fig. 3,a). This behavior is typical for microwave enhanced phase diffusion, as it has been discussed in Ref. 6. In particular, we observed for such a regime $V_{m} \propto P^{1 / 2}$.

As the temperature is decreased and for moderate values of the microwave power we observed the crossover to the resistive state characterized by a negative resistance at the bottom of the zero-crossing step (see Fig. 3,b,c). A voltage position of the zero-crossing step shifts to high 


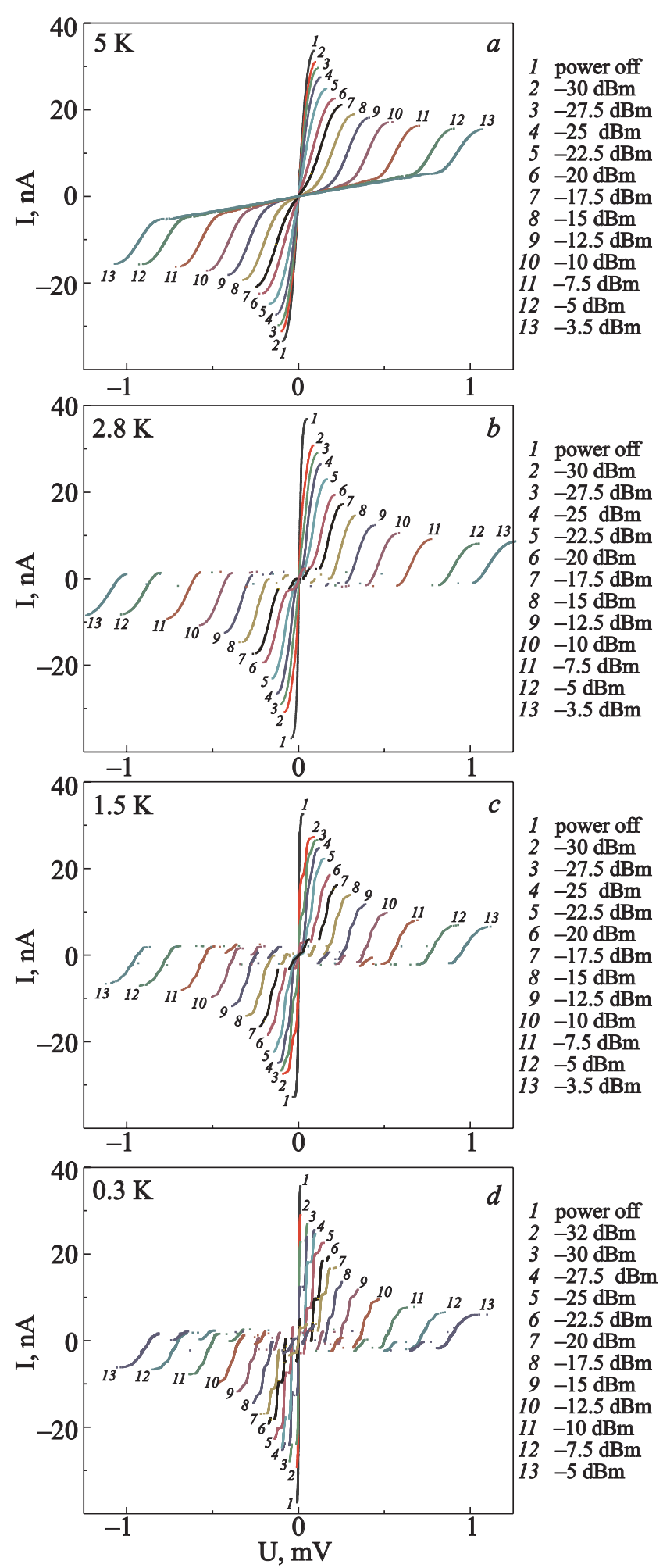

Fig. 3. The dependence of $I-V$ curves (the low voltage part is shown only) of a small Josephson junction on the power of microwave radiation. The $I-V$ curves are measured at different temperatures $T$, K: $5(a) ; 2.8(b) ; 1.5(c) ; 0.3(d)$. The area of Josephson junction is $0.28 \times 0.28 \mu \mathrm{m}$ and the frequency of microwave radiation is $\omega=20 \mathrm{GHz}$. Different colors correspond to the different power of microwave radiation. voltages with the power of microwave radiation. Moreover, as the temperature is decreased further such a state splits in several equally spaced steps (see Fig. 3,d). These steps are tilted and the slope increases with the rising microwave power. The distance between these steps is close to the value, of the distance between Shapiro steps in a Josephson junction under microwave radiation, i.e., $40-41 \mu \mathrm{V}$ for the chosen microwave frequency. Thus, a variation of temperature leads to an interesting crossover from incoherent to coherent microwave induced low-voltage resistive states of a small Josephson junction.

A similar crossover between different resistive states is observed as we change the frequency of microwave radiation $\omega$ or sample size while keeping the temperature constant. To study that we fixed temperature at $T=4.2 \mathrm{~K}$ and varied $\omega$ from $40 \mathrm{GHz}$ down to $1 \mathrm{GHz}$. These results are presented in Fig. 4 for the Josephson junction with area $0.47 \times 0.47 \mu \mathrm{m}$. It is found that at $40 \mathrm{GHz}$, the low-voltage branch displays a zero-crossing (see Fig. 4,a). Moreover, a phase diffusion branch tends to split in Shapiro steps, in the way similar to the described above for low temperatures (compare, Fig. 3, $d$ and Fig. 4,a). However, this effect is not strongly pronounced. As the frequency is reduced to $10 \mathrm{GHz}$, the $I-V$ curves do not show any sign of the splitting effect, i.e., the $I-V$ curves are smooth and, at the same time, they are zero-crossing in a wide range of power, as it is shown in Fig. 4,b. A decrease of the microwave frequency to $5 \mathrm{GHz}$ causes the zero-crossing phenomenon to disappear completely (see Fig. 4,c). Further decrease of the frequency makes the effect of zero-crossing to appear again (see Fig. 4,d).

The area (and thus the Josephson energy $E_{J}$ ) of the junctions also influences significantly on the appearance of different microwave induced low-voltage resistive states. Indeed for the frequency $\omega=40 \mathrm{GHz}$ and at temperature $T=4.2 \mathrm{~K}$ we observed that the junction of the area $0.23 \times 0.23 \mu \mathrm{m}$ displays a weak zero-crossing. As the area is increased the substantially more pronounced effect of a zero-crossing is found (see Fig. 4,a). However, further increase of the junctions area makes the zero-crossing effect less pronounced for a sample $1.1 \times 1.1 \mu \mathrm{m}$, and the zero crossing state completely vanished for a junction $2.05 \times 2.05 \mu \mathrm{m}$.

For a sample of moderate size we found also that the microwave radiation of a high frequency leads not only to the zero-crossing state but also to a fine structure of a lowvoltage resistive branch. Indeed, we observed up to 4 intermediate branches for a sample of moderate size $0.5 \times 0.5 \mu \mathrm{m}$. These results are presented in Fig. 5,a. It is interested that the voltage distance between the fine branches $\Delta U$ does not change with the microwave power resembling in this way the Shapiro steps. However, it is clear that the value of $\Delta U$ is by factor of two or more larger than the one corresponding to Shapiro steps. The voltage positions of these additional branches shift to higher values 

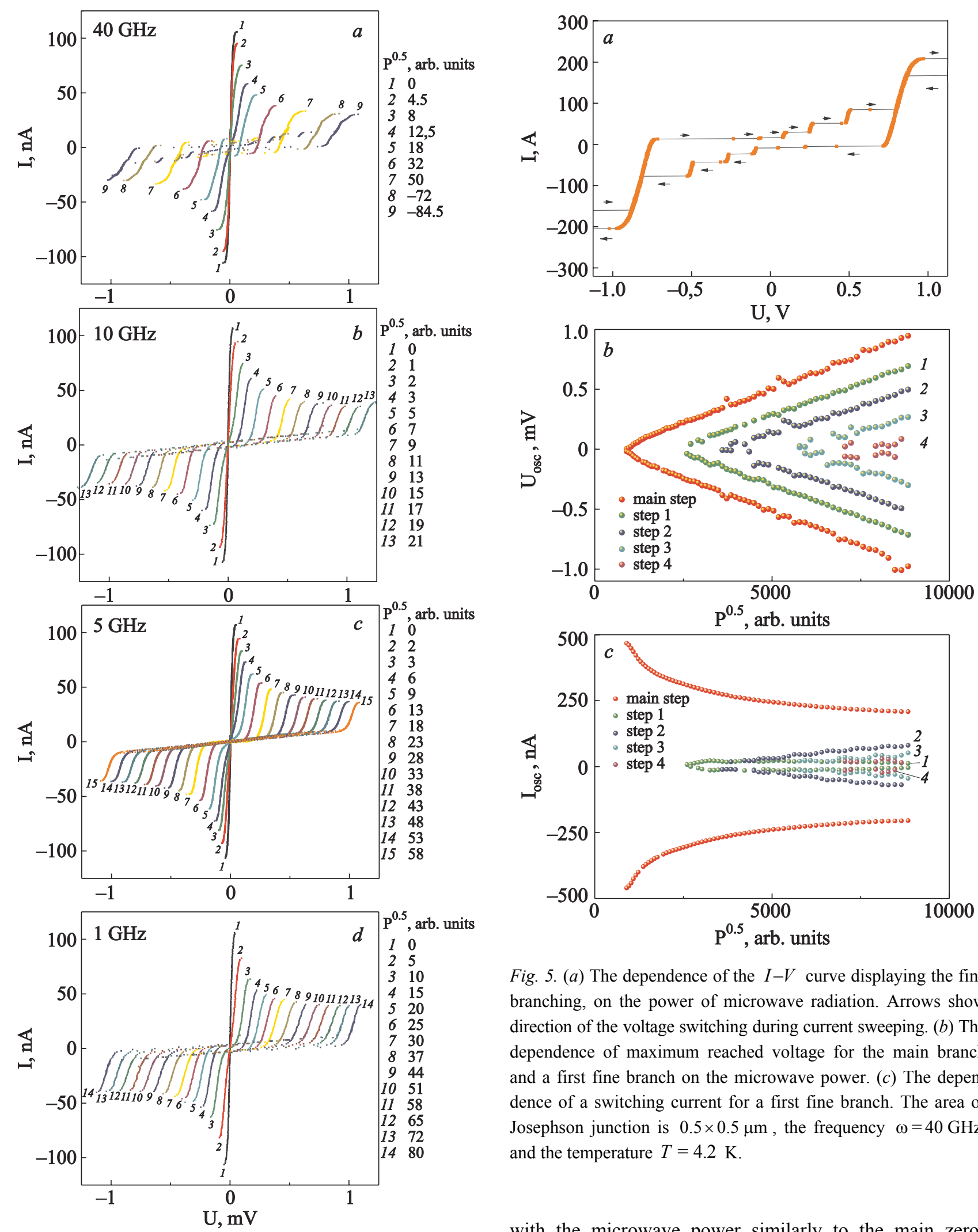

Fig. 5. (a) The dependence of the $I-V$ curve displaying the fine branching, on the power of microwave radiation. Arrows show direction of the voltage switching during current sweeping. (b) The dependence of maximum reached voltage for the main branch and a first fine branch on the microwave power. (c) The dependence of a switching current for a first fine branch. The area of Josephson junction is $0.5 \times 0.5 \mu \mathrm{m}$, the frequency $\omega=40 \mathrm{GHz}$, and the temperature $T=4.2 \mathrm{~K}$.

with the microwave power similarly to the main zerocrossing step. In Fig. 5,b we show the dependence of the maximum voltage for the main low-voltage resistive state and all intermediate branches on power of radiation. On contrary the maximum reached current of the intermediate branches changes in a different way in comparison to the main branch (see Fig. 5,c). The maximum reached currents increase with microwave power. Moreover, small oscilla-

Fig. 4. The dependence of $I-V$ curves (the low voltage part is shown only) of a small Josephson junction on the power of microwave radiation. The $I-V$ curves are measured at different frequencies of microwave radiation $\omega, \mathrm{GHz}: 40(a) ; 10(b)$; $5(c) ; 1(d)$. The area of Josephson junction is $0.47 \times 0.47 \mu \mathrm{m}$ and the temperature was fixed at $T=4.2 \mathrm{~K}$. Different colors correspond to the different power of microwaves. 
tions of these currents are detected. We stress here that the intermediate branches can be observed only for the conditions when the main branch displays a zero-crossing step.

The response to the microwave radiation similar to observed for $\mathrm{Nb}$ junctions is found also for intrinsic Josephson junctions in high-temperature superconductors BSCCO (2212). This material contains alternating superconducting and insulating layers. Thus, it is a well known that the transport in the transverse direction is determined by the Josephson effect. We made experiments with intrinsic Josephson junctions [11] of area $0.5 \times 0.5 \mu \mathrm{m}$. The measurements are fulfilled at $4.2 \mathrm{~K}$. Nominal critical current density of the used material is $1000 \mathrm{~A} / \mathrm{cm}^{2}$. The nominal critical current is $I_{c}=2.5 \mu \mathrm{A}$.

The microwave induced current-voltage characteristics are presented in Fig. 6. In the absence of the microwave radiation we observed the low-voltage resistive branch, switching to the high voltage region and hysteretic behavior. However, the finite slope of a low-voltage resistive branch is most probably due to a finite contact resistance between the top Au electrode and the top superconducting electrode of a Josephson junction as it was discussed previously. Because all essential parameters of our sample are
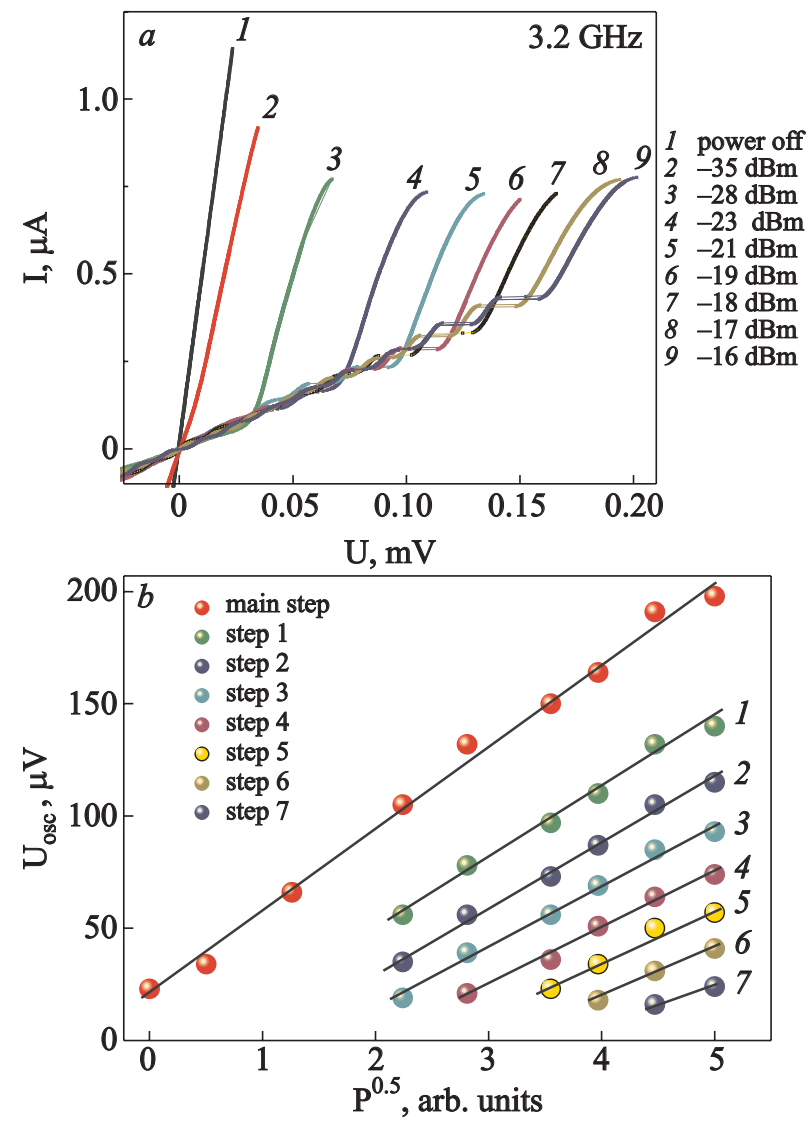

Fig. 6. The $I-V$ curves of BSCCO (2212) material containing intrinsic Josephson junctions at different values of microwave power. Area of the BSCCO sample is $0.5 \times 0.5 \mu \mathrm{m}$. Frequency of radiation is $3.2 \mathrm{GHz}$. Measurements are fulfilled at temperature $4.2 \mathrm{~K}$. similar to the ones of a $\mathrm{Nb}$ based Josephson junction of area $1 \times 1 \mu \mathrm{m}$ we conclude that the Josephson coupling energy is high enough and no Josephson phase diffusion is observed without microwave radiation. However, in the presence of microwave radiation the critical current of the Josephson junction was suppressed and the phase diffusion branch develops as it should be for microwave radiation enhanced phase diffusion. The maximum reached voltage $V_{m}$ of the low-voltage resistive state branch varies linearly with $P^{1 / 2}$.

A fine branching is also easily observed in BSCCO samples in spite of the fact that the main branch does not display a zero crossing.

\section{Microwave induced current-voltage characteristics: theory}

Next we present our theoretical analysis of various microwave induced low-voltage resistive states of a small Josephson junction biased in Josephson phase diffusion regime. A small Josephson junction is characterized by the Josephson phase $\varphi(t)$ satisfying the equation:

$$
\ddot{\varphi}(t)+\alpha \dot{\varphi}(t)+\sin \varphi(t)=j+\eta \sin \omega t+\xi(t) .
$$

In this equation, $j$ is an external dc bias, $\eta$ and $\omega$ are amplitude and frequency of an external microwave radiation, $\xi(t)$ is a random function of time $t$ describing thermal fluctuations. Here, we use dimensionless units, i.e., the time is normalized to $\omega_{p}^{-1}$, the dc bias is normalized to the critical current value $I_{c}$. The dissipative effects are described by parameter $\alpha$ that, in general, can be frequency dependent. The presence of hysteretic regime in the observed $I-V$ curves allows us to propose a simple model, assuming that the low frequency damping $\alpha_{0}$ determined by the quasiparticle current is weak $\left(\alpha_{0} \ll 1\right)$ but the high frequency damping $\alpha$ is large. As the Josephson coupling energy $E_{\mathrm{J}}$ is comparable with the energy of thermal fluctuations $k_{B} T$, i.e., the Josephson phase diffusion is strong, the incoherent superconducting current can be found from the following analysis. In the absence of applied microwave radiation the Josephson phase is written as

$$
\varphi(t)=v t+\psi(t)+\varphi_{1}(t),
$$

where

$$
\psi(t)=\frac{1}{\alpha} \int_{0}^{t} \xi(t) d t
$$

determines the Josephson phase diffusion. Here, the dc voltage drop across the Josephson junction $V$ is normalized to

$$
V_{p}=\frac{\Phi_{0} \omega_{p}}{2 \pi}=\frac{1}{2 e} \sqrt{8 E_{\mathrm{J}} E_{\mathrm{C}}}
$$

as $v=V / V_{p}$. In this case, the term $\varphi_{1}(t)$ is expressed through the alternating part of the superconducting current 


$$
\varphi_{1}(t)=\frac{1}{\alpha} \int_{0}^{t} d t_{1} \sin \left(v t_{1}+\psi\left(t_{1}\right)\right) .
$$

The dc component of the incoherent superconducting current is found as

$$
\begin{gathered}
I_{s}^{0}(v)=\frac{I_{c}}{\alpha} \lim _{T \rightarrow \infty} \int_{0}^{T} \frac{d t}{T} \cos (v t+\psi(t)) \int_{0}^{t} d t_{1} \sin \left(v t_{1}+\psi\left(t_{1}\right)\right)= \\
\quad=\frac{I_{c}}{\alpha} \int_{0}^{\infty} d t \rho(t) \sin (v t)
\end{gathered}
$$

where $\rho(t)=<\cos (\psi(t)-\psi(0))>$ is the correlation function of a random part of the Josephson phase. This correlation function has been studied in detail for different models in $[4,12]$ and, in the simplest case of Gaussian random function $\xi(t)$, the $\rho(t)$ has a diffusive form $\rho(t)=\exp (-\delta t)$. Substituting this expression into (4) we obtain $[12,13]$

$$
I_{s}^{0}=\frac{I_{c}}{\alpha} \frac{v}{v^{2}+\delta^{2}}=\frac{I_{c}}{\alpha} \frac{V V_{p}}{V^{2}+\left(\delta V_{p}\right)^{2}}
$$

Thus, the Josephson phase diffusion regime combined with the large high-frequency and small low-frequency damping, leads to a strongly nonlinear current-voltage characteristics. In the presence of microwave radiation the Josephson phase can be written as

$$
\varphi(t)=v t+\psi(t)-\frac{\eta}{\alpha} \cos \omega t+\varphi_{1}(t) .
$$

Applying the procedure that is similar to the above treatment of the Josephson phase diffusion without microwave radiation, we obtain the expression for incoherent superconducting current.

$$
I_{s}=\sum_{n=-\infty}^{\infty} J_{n}^{2}\left(\frac{\eta}{\alpha \omega}\right) I_{s}^{0}(v-n \omega),
$$

where $J_{n}(x)$ is the Bessel function of index $n$, and $n$ is an integer number. Notice here, that equation (7) allows one to express the dc current in the presence of microwave radiation through the autonomous $I-V$ curve obtained previously for diverse physical systems [14], e.g. quasiparticle current in superconducting tunnel junctions, Coulomb blockade regime, semiconducting superlattices. The generic character of Eq. (7) describes the process of incoherent multiphoton absorption in a strongly nonlinear system with $n$ being the number of photons.

In the following we numerically calculate the resulting $I-V$ curve for various combination of parameters. Although our analysis is valid in the limit of a strong phase diffusion, i.e. as $E_{J} \leq k_{B} T$, in the following qualitative discussion we will use Eqs. (5) and (7) considering the $\delta$ and $\alpha$ as fitting parameters. First, in the limit of a low

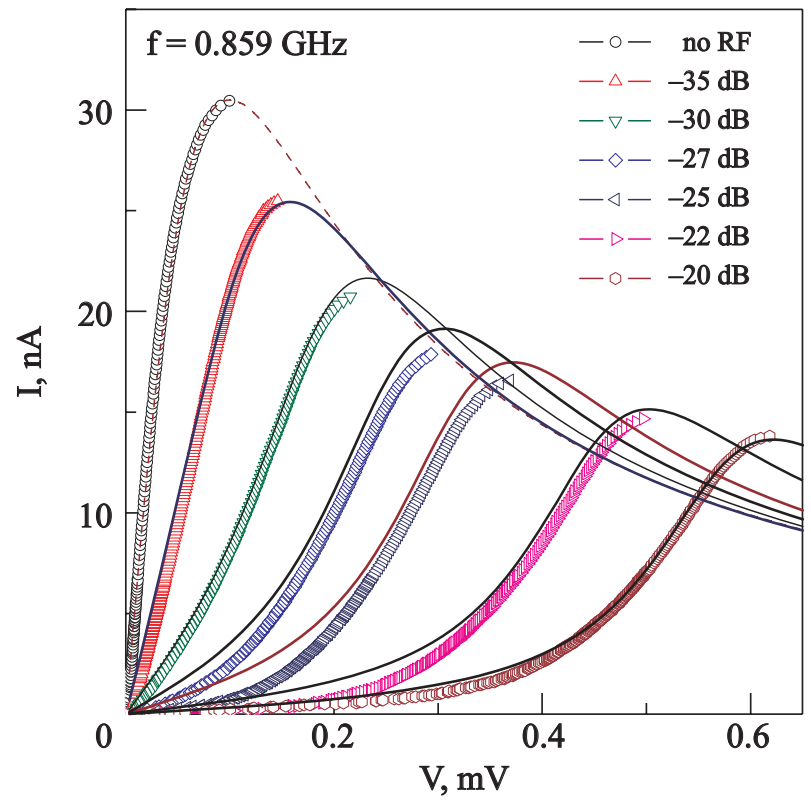

Fig. 7. The numerically calculated $I-V$ curves (Eqs. (7) and (5)) for different values of microwave power: enhanced phase diffusion regime. The parameter $\delta / \omega=5$ is chosen [6].

frequency $\omega$ or more precisely as $\omega \ll V_{m}=\delta V_{p}$ we obtain the regime of an enhanced phase diffusion. This regime has been described in detail in Ref. 6, and the dependence of $I-V$ curves on microwave power is presented in Fig. 7. As the microwave frequency increases, or the Josephson phase diffusion becomes weaker, and therefore, the characteristic voltage $V_{m}$ decreases and we obtain the zero-crossing step and the regions with negative resistance (see Fig. 8). When comparing this theoretical curve to the experimental data we should note that in experiment due to the use current-biasing mode the regions of $I-V$ curves with negative differential resistance (negative slope) were

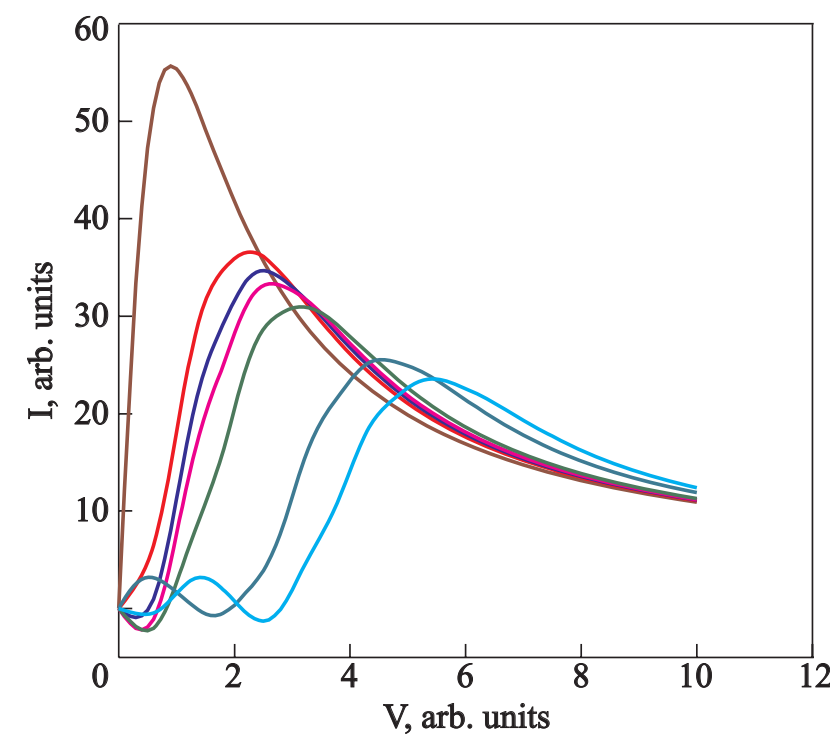

Fig. 8. The numerically calculated $I-V$ curves (Eqs. (7) and (5)) for different values of microwave power: «zero crossing step» regime. The parameter $\delta / \omega=0.9$ is chosen. 


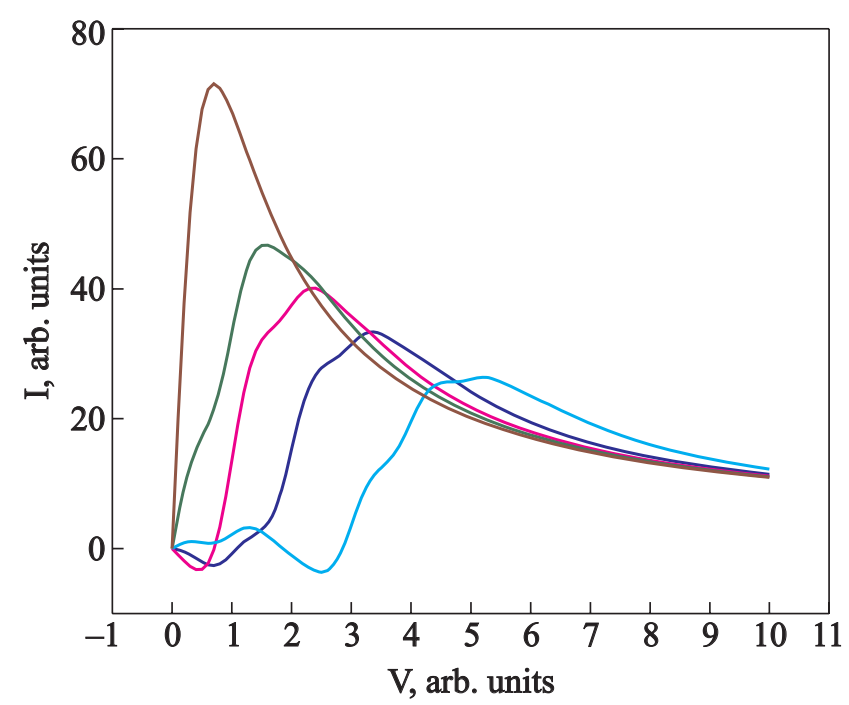

Fig. 9. The numerically calculated $I-V$ curves (Eqs. (7) and (5)) for different values of microwave power showing the fine branching state regime. The parameters $\delta / \omega=0.7$ are chosen here.

not stable and thus not observed. Finally, the further frequency increase leads to a fine branching state at the $I-V$ curve (see Fig. 9).

\section{Discussion and conclusions}

All our experimental results on microwave induced low-voltage resistive states can be directly compared with the theoretical $I-V$ curves derived in the previous section. The analysis is carried out in the model for a process of incoherent absorption in a small Josephson junction biased in the Josephson phase diffusion regime. Moreover, we obtained that the resulting microwave induced lowvoltage resistive state is determined by the ratio of the photon energy $\hbar \omega$ to the maximum reached voltage $V_{m}$ characterizing the Josephson phase diffusion process. This ratio can be tuned by different methods, e.g. directly by the frequency change or indirectly by change of a junction area or temperature. Indeed, as this ratio $\hbar \omega / V_{m}$ is small the regime of an enhanced phase diffusion is obtained (compare experimental Figs. 3,a, 4,c, and 6 with the theoretical Fig. 7). The increase of this ratio leads to a «ze-ro-crossing step» state characterizing by a negative resistance (see Figs. 3, $b-d, 4, a, b$, and Fig. 8). Such a microwave induced resistive state is a particular example of an intriguing ratchet effect as the Josephson phase dynamics displays a temporal broken symmetry in the absence of directed force. Notice here, that the necessary condition for the observation of a zero-crossing step in a small Josephson junctions is a large quasiparticle resistance. We argue that it is a reason why the zero-crossing step is not observed in our experiments with high-temperature superconductor based BSCCO junction stack (see Fig. 6). A larger value of photon energy $\hbar \omega$ allows to resolve single Shapiro steps (see
Fig. 3,d). A further increase of $\hbar \omega / V_{m}$ ratio leads to a fine branching state (see Fig. 5 and Fig. 9).

In conclusion, we experimentally observed the various low-voltage resistive states of small (micrometer-scale) Josephson junctions. These states appear in the presence of a strong microwave irradiation. The crossover between different regimes is found as the frequency, temperature and /or size of the junction vary. Similar states were observed in submicron-area mesas of high-temperature superconductors consisting of a stack of Josephson coupled layers. We proposed a specific model of incoherent multiphoton absorption by a small Josephson junction biased in the Josephson phase diffusion regime. Our analysis shows that the appearance of different state is determined by the ratio $\hbar \omega / V_{m}$, and this analysis is in a good accord with experimental observations.

As the bottom line, we would like to mention that similar behavior to the microwave-enhanced incoherent phase diffusion can be found in different Josephson coupled system, namely long Josephson junctions where the $I-V$ curve is determined by motion of Josephson fluxons. Indeed, the enhanced phase diffusion in the form of stochastic fluxon motion has been found in Ref. 15. Moreover, a zero-crossing step has been found in numerical calculations of such system [16]. It can be argued that the stochastic character of the fluxon motion qualitatively plays a role here that mimics thermal fluctuations and thus leads to similar consequences.

M.V.F. acknowledges the financial support by SFB 491. This work was supported by the DFG-Center for Functional Nanostructures (CFN) and the EU project SCOPE.

1. M.H. Devoret, J.M. Martinis, and J. Clarke, Phys. Rev. Lett. 55, 1908 (1985); J.M. Martinis, M.H. Devoret, and J. Clarke, Phys. Rev. B35, 4682 (1987).

2. D.V. Averin, A.B. Zorin, and K.K. Likharev, Sov. Phys. JETP 61, 407 (1985); D.V. Averin, and K.K. Likharev, J. Low Temp. Phys. 62, 345 (1986).

3. J.M. Martinis and R.L. Kautz, Phys. Rev. Lett. 63, 1507 (1989); R.L. Kautz, and J.M. Martinis, Phys. Rev. B42, 9903 (1990).

4. G.-L. Ingold, H. Grabert, and U. Eberhardt, Phys. Rev. B50, 395 (1994).

5. D. Vion, M. Götz, P. Joyez, D. Esteve, and M.H. Devoret, Phys. Rev. Lett. 77, 3435 (1996).

6. Y. Koval, M.V. Fistul, and A.V. Ustinov, Phys. Rev. Lett. 93, 087004 (2004).

7. B.J. Keay, S.J. Allen, Jr., J. Galán, J.P. Kaminski, K.L. Campman, A.C. Gossard, U. Bhattacharya, and M.J.W. Rodwel, Phys. Rev. Lett. 75, 4098 (1995).

8. R.G. Mani, J.H. Smet, K. von Klitzing, V. Narayannmurti, W.B. Johnson, and V. Umansky, Nature (London) 420, 646 (2002); M.A. Zudov, R.R. Du, L.N. Pfeiffer, and K.W. West, Phys. Rev. Lett. 90, 046807 (2003). 
9. S. Shapiro, Phys. Rev. Lett. 11, 80 (1963).

10. Y. Koval, A. Wallraff, M. Fistul, N. Thyssen, H. Kohlstedt, and A.V. Ustinov, IEEE Trans. Appl. Supercond. 9, 3957 (1999).

11. A. Franz, Y. Koval, D. Vasyukov, P. Müller, H. Schneidewind, S.A. Ryndyk, J. Keller, and C. Helm, Phys. Rev. B69, 014506 (2004).

12. G.-L. Ingold and Yu.V. Nazarov, in: Single Charge Tunneling, H. Grabert and M.H. Devoret (eds.), NATO ASI, Ser. B, Vol. 294, Plenum, New York (1991).
13. M. Tinkham, Introduction to Superconductivity, McGrawHill, New York (1996).

14. Equation (7) was obtained by G. Falci, V. Bubanja, and G. Schön, Z. Phys. B85, 451 (1991).

15. F.L. Barkov, M.V. Fistul, and A.V. Ustinov, Phys. Rev. B70, 134515 (2004).

16. E. Goldobin, B.A. Malomed, and A.V. Ustinov, Phys. Rev. E65, 056613 (2002). 